\title{
PERIODIC AND ASYMPTOTICALLY PERIODIC SOLUTIONS FOR NEUTRAL NONLINEAR COUPLED VOLTERRA INTEGRO-DIFFERENTIAL SYSTEMS WITH TWO VARIABLE DELAYS
}

\author{
BOUZID MANSOURI ${ }^{1,2, a}$, ABDELOUAHEB ARDJOUNI ${ }^{3,4, b, *}$ \\ AND AHCENE DJOUDI ${ }^{5, c}$
}

\footnotetext{
${ }^{1}$ Faculty of Sciences, Department of Mathematics, Univ Annaba, P.O. Box 12, Annaba, 23000, Algeria.

${ }^{2}$ Technical and Teacher Training School, Skikda, 21000, Algeria.

${ }^{a}$ E-mail: mansouri.math@yahoo.fr

${ }^{3}$ Faculty of Sciences and Technology, Department of Mathematics and Informatics, Univ Souk Ahras, P.O. Box 1553, Souk Ahras, 41000, Algeria.

${ }^{4}$ Applied Mathematics Lab, Faculty of Sciences, Department of Mathematics, Univ Annaba, P.O. Box 12, Annaba 23000, Algeria.

${ }^{b}$ E-mail: abd_ardjouni@yahoo.fr

${ }^{5}$ Applied Mathematics Lab, Faculty of Sciences, Department of Mathematics, Univ Annaba, P.O. Box 12, Annaba 23000, Algeria.

${ }^{c}$ E-mail: adjoudi@yahoo.com
}

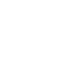

\section{Abstract}

In this paper, we study the existence of periodic and asymptotically periodic solutions of neutral nonlinear coupled Volterra integro-differential systems. We furnish sufficient conditions for the existence of such solutions. Krasnoselskii's fixed point theorem is used in this analysis.

\section{Introduction}

The study of the existence of periodic solutions is one of the most interesting and important topics in the qualitative theory of differential equations. Some contributions on the existence of periodic solutions for differential equations have been made (see [1]-[4], [19], [20]). On the other hand,

Received December 12, 2021.

AMS Subject Classification: 34K20, 45J05, 45D05.

Key words and phrases: Volterra integro-differential equations, neutral differential equations, periodic solutions, asymptotic periodic solutions, Krasnoselskii fixed point theorem.

*Corresponding Author. 
the concept of asymptotic periodicity is more general than periodicity and from an applied perspective, asymptotically periodic systems describe world more realistically and accurately than periodic ones, we can see [8], [9], [12], [26], [27], for more details.

In 1928 Volterra [25] noted that many physical problems were being modeled by integral and integro-differential equations. Today we see that such models have application in several branches of applied science, such as control theory, mathematical biology, viscoelasticity, nuclear reactors, many other areas, and for this reason this type of equation has received much attention in recent years, (see for example [5], [7], [10], [11], [14]-[21], [23]). Motivated by the papers [6], [13], [22] and the references therein and by using Krasnoselskii's fixed point theorem, in this paper, we study the existence of periodic and asymptotically periodic solutions of the following system of coupled neutral nonlinear Volterra integro-differential equations with two delays

$$
\left\{\begin{aligned}
x^{\prime}(t)= & h_{1}(t) x(t)+G_{1}\left(t, x(t), y(t), x\left(t-\tau_{1}(t)\right), y\left(t-\tau_{2}(t)\right)\right) \\
& +c_{1}(t) x^{\prime}\left(t-\tau_{1}(t)\right)+\int_{-\infty}^{t} a_{1}(t, s) f_{1}(x(s), y(s)) d s \\
y^{\prime}(t)= & h_{2}(t) y(t)+G_{2}\left(t, x(t), y(t), x\left(t-\tau_{1}(t)\right), y\left(t-\tau_{2}(t)\right)\right) \\
& +c_{2}(t) y^{\prime}\left(t-\tau_{2}(t)\right)+\int_{-\infty}^{t} a_{2}(t, s) f_{2}(x(s), y(s)) d s
\end{aligned}\right.
$$

where the functions $h_{i}, c_{i}$ and $a_{i}, i=1,2$ are assumed to be continuous in their arguments throughout the paper. The functions $G_{i}(t, x, y, z, w), i=$ 1,2 is continuous, periodic in $t$ and Lipschitz continuous in $x, y, z$ and $w$, $f_{i}(x, y), i=1,2$ is continuous and Lipschitz continuous in $x$ and $y$, and for some positive constants $N_{j}$ and $R_{j}, j=\overline{1,4}$ we have

$$
\begin{aligned}
& \left|G_{1}\left(t, y_{1}, y_{2}, y_{3}, y_{4}\right)-G_{1}\left(t, x_{1}, x_{2}, x_{3}, x_{4}\right)\right| \leq \sum_{j=1}^{4} N_{j}\left|y_{j}-x_{j}\right| \\
& \left|G_{2}\left(t, y_{1}, y_{2}, y_{3}, y_{4}\right)-G_{2}\left(t, x_{1}, x_{2}, x_{3}, x_{4}\right)\right| \leq \sum_{j=1}^{4} R_{j}\left|y_{j}-x_{j}\right|
\end{aligned}
$$

and for some positive constants $d_{j}$ and $q_{j}, j=1,2$ we have

$$
\left|f_{1}\left(y_{1}, y_{2}\right)-f_{1}\left(x_{1}, x_{2}\right)\right| \leq \sum_{j=1}^{2} d_{j}\left|y_{j}-x_{j}\right|,
$$




$$
\left|f_{2}\left(y_{1}, y_{2}\right)-f_{2}\left(x_{1}, x_{2}\right)\right| \leq \sum_{j=1}^{2} q_{j}\left|y_{j}-x_{j}\right|
$$

we also assume that $G_{1}(t, 0,0,0,0)=G_{2}(t, 0,0,0,0)=f_{1}(0,0)=f_{2}(0,0)=$ 0 .

We assume that there exists a positive real number $T$, such that

$$
\begin{aligned}
h_{i}(t+T) & =h_{i}(t), c_{i}(t+T)=c_{i}(t), \\
a_{i}(t+T, s+T) & =a_{i}(t, s), \tau_{i}(t+T)=\tau_{i}(t), \quad i=1,2,
\end{aligned}
$$

with $c_{i}$ continuously differentiable, $\tau_{i}$ twice continuously differentiable and $\tau_{i}(t) \geq \tau_{i}^{*}>0$ for $i=1,2$. To have a well behaved mapping we must assume that

$$
\tau_{i}^{\prime}(t) \neq 1, \int_{0}^{T} h_{i}(s) d s \neq 0, i=1,2 .
$$

Define $P_{T}=\{(\varphi, \psi):(\varphi, \psi)(t+T)=(\varphi, \psi)(t)\}$, where both $\varphi$ and $\psi$ are real valued continuous functions on $\mathbb{R}$. Then $P_{T}$ is a Banach space when endowed with the maximum norm

$$
\|(x, y)\|=\max \left\{\max _{t \in[0, T]}|x(t)|, \max _{t \in[0, T]}|y(t)|\right\} .
$$

Definition 1.1. A function $x$ is called asymptotically $T$-periodic if there exist two functions $x_{1}$ and $x_{2}$ such that $x_{1}$ is $T$-periodic, $\lim _{t \rightarrow \infty} x_{2}(t)=0$ and $x(t)=x_{1}(t)+x_{2}(t)$ for all $t$.

Lemma 1.2. Assume (1.2) and (1.3). If $x, y \in P_{T}$, then $x$ and $y$ is a solution of (1.1) if and only if

$$
\begin{aligned}
x(t)= & \frac{c_{1}(t) x\left(t-\tau_{1}(t)\right)}{1-\tau_{1}^{\prime}(t)}-\int_{t}^{t+T} \frac{e^{\int_{u}^{t+T} h_{1}(s) d s}}{1-e^{\int_{0}^{T} h_{1}(s) d s}} r_{1}(u) x\left(u-\tau_{1}(u)\right) d u \\
& +\int_{t}^{t+T} \frac{e^{\int_{u}^{t+T} h_{1}(s) d s}}{1-e^{\int_{0}^{T} h_{1}(s) d s}} G_{1}\left(u, x(u), y(u), x\left(u-\tau_{1}(u)\right), y\left(u-\tau_{2}(u)\right)\right) d u \\
& +\int_{t}^{t+T} \frac{e^{\int_{u}^{t+T} h_{1}(s) d s}}{1-e^{\int_{0}^{T} h_{1}(s) d s}} \int_{-\infty}^{u} a_{1}(u, s) f_{1}(x(s), y(s)) d s d u
\end{aligned}
$$

and 


$$
\begin{aligned}
y(t)= & \frac{c_{2}(t) y\left(t-\tau_{2}(t)\right)}{1-\tau_{2}^{\prime}(t)}-\int_{t}^{t+T} \frac{e^{\int_{u}^{t+T} h_{2}(s) d s}}{1-e^{\int_{0}^{T} h_{2}(s) d s}} r_{2}(u) y\left(u-\tau_{2}(u)\right) d u \\
& +\int_{t}^{t+T} \frac{e^{\int_{u}^{t+T} h_{2}(s) d s}}{1-e^{\int_{0}^{T} h_{2}(s) d s}} G_{2}\left(u, x(u), y(u), x\left(u-\tau_{1}(u)\right), y\left(u-\tau_{2}(u)\right)\right) d u \\
& +\int_{t}^{t+T} \frac{e^{\int_{u}^{t+T} h_{2}(s) d s}}{1-e^{\int_{0}^{T} h_{2}(s) d s}} \int_{-\infty}^{u} a_{2}(u, s) f_{2}(x(s), y(s)) d s d u
\end{aligned}
$$

where

$$
r_{1}(u)=\frac{\left(c_{1}^{\prime}(u)-c_{1}(u) h_{1}(u)\right)\left(1-\tau_{1}^{\prime}(u)\right)+\tau_{1}^{\prime \prime}(u) c_{1}(u)}{\left(1-\tau_{1}^{\prime}(u)\right)^{2}},
$$

and

$$
r_{2}(u)=\frac{\left(c_{2}^{\prime}(u)-c_{2}(u) h_{2}(u)\right)\left(1-\tau_{2}^{\prime}(u)\right)+\tau_{2}^{\prime \prime}(u) c_{2}(u)}{\left(1-\tau_{2}^{\prime}(u)\right)^{2}} .
$$

Proof. Let $x, y \in P_{T}$ be a solution of (1.1). Next we multiply both sides of the first equation in (1.1) by $e^{-\int_{0}^{t} h_{1}(s) d s}$ and then integrate from $t$ to $t+T$, to obtain

$$
\begin{aligned}
& \int_{t}^{t+T}\left[x(u) e^{\left.-\int_{0}^{u} h_{1}(s) d s\right]^{\prime} d u}\right. \\
& =\int_{t}^{t+T} e^{-\int_{0}^{u} h_{1}(s) d s} G_{1}\left(u, x(u), y(u), x\left(u-\tau_{1}(u)\right), y\left(u-\tau_{2}(u)\right)\right) d u \\
& \quad+\int_{t}^{t+T} e^{-\int_{0}^{u} h_{1}(s) d s} c_{1}(u) x^{\prime}\left(u-\tau_{1}(u)\right) d u \\
& \quad+\int_{t}^{t+T} e^{-\int_{0}^{u} h_{1}(s) d s} \int_{-\infty}^{u} a_{1}(u, s) f_{1}(x(s), y(s)) d s d u .
\end{aligned}
$$

Consequently, we have

$$
\begin{aligned}
& x(t+T) e^{-\int_{0}^{t+T} h_{1}(s) d s}-x(t) e^{-\int_{0}^{t} h_{1}(s) d s} \\
& =\int_{t}^{t+T} e^{-\int_{0}^{u} h_{1}(s) d s} G_{1}\left(u, x(u), y(u), x\left(u-\tau_{1}(u)\right), y\left(u-\tau_{2}(u)\right)\right) d u \\
& \quad+\int_{t}^{t+T} e^{-\int_{0}^{u} h_{1}(s) d s} c_{1}(u) x^{\prime}\left(u-\tau_{1}(u)\right) d u \\
& \quad+\int_{t}^{t+T} e^{-\int_{0}^{u} h_{1}(s) d s} \int_{-\infty}^{u} a_{1}(u, s) f_{1}(x(s), y(s)) d s d u .
\end{aligned}
$$


Multiply both sides with $e^{\int_{0}^{t+T} h_{1}(s) d s}$ and using the fact that $x(t+T)=x(t)$ and $e^{\int_{t}^{t+T}} h_{1}(s) d s=e^{\int_{0}^{T} h_{1}(s) d s}$, we obtain

$$
\begin{aligned}
x(t)= & \int_{t}^{t+T} \frac{e^{\int_{u}^{t+T} h_{1}(s) d s}}{1-e^{\int_{0}^{T} h_{1}(s) d s}} G_{1}\left(u, x(u), y(u), x\left(u-\tau_{1}(u)\right), y\left(u-\tau_{2}(u)\right)\right) d u \\
& +\int_{t}^{t+T} \frac{e^{\int_{u}^{t+T} h_{1}(s) d s}}{1-e^{\int_{0}^{T} h_{1}(s) d s}} c_{1}(u) x^{\prime}\left(u-\tau_{1}(u)\right) d u \\
& +\int_{t}^{t+T} \frac{e^{\int_{u}^{t+T}} h_{1}(s) d s}{1-e^{\int_{0}^{T} h_{1}(s) d s}} \int_{-\infty}^{u} a_{1}(u, s) f_{1}(x(s), y(s)) d s d u .
\end{aligned}
$$

Letting

$$
\begin{aligned}
& \int_{t}^{t+T} e^{\int_{u}^{t+T} h_{1}(s) d s} c_{1}(u) x^{\prime}\left(u-\tau_{1}(u)\right) d u \\
& \quad=\int_{t}^{t+T} \frac{e^{\int_{u}^{t+T} h_{1}(s) d s} c_{1}(u)}{1-\tau_{1}^{\prime}(u)}\left(1-\tau_{1}^{\prime}(u)\right) x^{\prime}\left(u-\tau_{1}(u)\right) d u .
\end{aligned}
$$

Performing an integration by parts, we get

$$
\begin{aligned}
& \int_{t}^{t+T} e^{\int_{u}^{t+T} h_{1}(s) d s} c_{1}(u) x^{\prime}\left(u-\tau_{1}(u)\right) d u \\
& =\left[\frac{c_{1}(u) x\left(u-\tau_{1}(u)\right)}{1-\tau_{1}^{\prime}(u)} e^{\int_{u}^{t+T} h_{1}(s) d s}\right]_{t}^{t+T}-\int_{t}^{t+T} e^{\int_{u}^{t+T} h_{1}(s) d s} r_{1}(u) x\left(u-\tau_{1}(u)\right) d u \\
& =\frac{c_{1}(t) x\left(t-\tau_{1}(t)\right)}{1-\tau_{1}^{\prime}(t)}\left(1-e^{\int_{0}^{T} h_{1}(s) d s}\right)-\int_{t}^{t+T} e^{\int_{u}^{t+T} h_{1}(s) d s} r_{1}(u) x\left(u-\tau_{1}(u)\right) d u,
\end{aligned}
$$

where $r_{1}$ is given by (1.6). Substituting (1.9) into (1.8), we obtain

$$
\begin{aligned}
x(t)= & \frac{c_{1}(t) x\left(t-\tau_{1}(t)\right)}{1-\tau_{1}^{\prime}(t)}-\int_{t}^{t+T} \frac{e^{\int_{u}^{t+T} h_{1}(s) d s}}{1-e^{\int_{0}^{T} h_{1}(s) d s}} r_{1}(u) x\left(u-\tau_{1}(u)\right) d u \\
& +\int_{t}^{t+T} \frac{e^{\int_{u}^{t+T} h_{1}(s) d s}}{1-e^{\int_{0}^{T} h_{1}(s) d s}} G_{1}\left(u, x(u), y(u), x\left(u-\tau_{1}(u)\right), y\left(u-\tau_{2}(u)\right)\right) d u \\
& +\int_{t}^{t+T} \frac{e^{\int_{u}^{t+T} h_{1}(s) d s}}{1-e^{\int_{0}^{T} h_{1}(s) d s}} \int_{-\infty}^{u} a_{1}(u, s) f_{1}(x(s), y(s)) d s d u .
\end{aligned}
$$

The proof of (1.5) is similar and hence we omit it. 


\section{Periodic Solutions}

Lemma 2.1 (24] ). Let $\mathbb{M}$ be a bounded closed convex nonempty subset of a Banach space $(S,\|\cdot\|)$. Suppose that $A$ and $B$ map $\mathbb{M}$ into $S$ such that

(i) $x, y \in \mathbb{M}$, implies $A x+B y \in \mathbb{M}$,

(ii) $A$ is compact and continuous,

(iii) $B$ is a contraction mapping.

Then there exists $z \in \mathbb{M}$ with $z=A z+B z$.

Let $\gamma_{i}(t)=\frac{c_{i}(t)}{1-\tau_{i}^{\prime}(t)}, i=1,2$, we assume that $\sup _{t \in[0, T]}\left|\gamma_{i}(t)\right|=\mu_{i}<1$, and let $\mu=\max \left\{\mu_{1}, \mu_{2}\right\}$. Let $\beta_{i}, i=1,2$ be positive constants such that $0<\mu_{i}+\beta_{i}<1$. Moreover, we assume the existence of positive constants $M_{i}, K_{i}, \alpha_{i}, L_{i}$ and $\theta_{i}, i=1,2$ such that

$$
\begin{gathered}
\left|f_{1}(x, y)\right| \leq M_{1}, \\
\left|f_{2}(x, y)\right| \leq M_{2}, \\
\left|G_{1}(t, x, y, z, w)\right| \leq K_{1},\left|G_{2}(t, x, y, z, w)\right| \leq K_{2}, \\
\int_{t}^{t+T}\left|\frac{e^{\int_{u}^{t+T} h_{1}(s) d s}}{1-e^{\int_{0}^{T} h_{1}(s) d s}}\right| \int_{-\infty}^{u}\left|a_{1}(u, s)\right| d s d u \leq \alpha_{1}, \\
\int_{t}^{t+T}\left|\frac{e^{\int_{u}^{t+T} h_{2}(s) d s}}{1-e^{\int_{0}^{T} h_{2}(s) d s}}\right| \int_{-\infty}^{u}\left|a_{2}(u, s)\right| d s d u \leq \alpha_{2}, \\
\int_{t}^{t+T}\left|\frac{e^{\int_{u}^{t+T} h_{1}(s) d s}}{1-e^{\int_{0}^{T} h_{1}(s) d s}}\right|\left|r_{1}(u)\right| d u \leq \beta_{1}, \\
\int_{t}^{t+T}\left|\frac{e^{\int_{u}^{t+T} h_{2}(s) d s}}{1-e^{\int_{0}^{T} h_{2}(s) d s}}\right|\left|r_{2}(u)\right| d u \leq \beta_{2}, \\
\int_{t}^{t+T}\left|\frac{e^{\int_{u}^{t+T} h_{1}(s) d s}}{1-e^{\int_{0}^{T} h_{1}(s) d s}}\right| d u \leq L_{1}, \int_{t}^{t+T}\left|\frac{e^{\int_{u}^{t+T} h_{2}(s) d s}}{1-e^{\int_{0}^{T} h_{2}(s) d s}}\right| d u \leq L_{2},
\end{gathered}
$$

and

$$
\int_{-\infty}^{u}\left|a_{1}(u, s)\right| d s \leq \theta_{1}, \quad \int_{-\infty}^{u}\left|a_{2}(u, s)\right| d s \leq \theta_{2} .
$$


Set

$$
M=\max \left\{\frac{L_{1} K_{1}+\alpha_{1} M_{1}}{1-\mu_{1}-\beta_{1}}, \frac{L_{2} K_{2}+\alpha_{2} M_{2}}{1-\mu_{2}-\beta_{2}}\right\} .
$$

We define subset $\Omega_{x, y}$ of $P_{T}$ as follows

$$
\Omega_{x, y}=\left\{(x, y):(x, y) \in P_{T} \text { with }\|(x, y)\| \leq M\right\}
$$

Then $\Omega_{x, y}$ is a bounded, closed and convex subset of $P_{T}$. Now for $(x, y) \in$ $\Omega_{x, y}$ we can define an operator $E: \Omega_{x, y} \rightarrow P_{T}$ by

$$
E(x, y)(t)=\left(E_{1}(x, y)(t), E_{2}(x, y)(t)\right)
$$

where

$$
\begin{aligned}
& E_{1}(x, y)(t) \\
& =\frac{c_{1}(t) x\left(t-\tau_{1}(t)\right)}{1-\tau_{1}^{\prime}(t)}-\int_{t}^{t+T} \frac{e^{\int_{u}^{t+T}} h_{1}(s) d s}{1-e^{\int_{0}^{T} h_{1}(s) d s}} r_{1}(u) x\left(u-\tau_{1}(u)\right) d u \\
& \quad+\int_{t}^{t+T} \frac{e^{\int_{u}^{t+T}} h_{1}(s) d s}{1-e^{\int_{0}^{T} h_{1}(s) d s}} G_{1}\left(u, x(u), y(u), x\left(u-\tau_{1}(u)\right), y\left(u-\tau_{2}(u)\right)\right) d u \\
& \quad+\int_{t}^{t+T} \frac{e^{\int_{u}^{t+T}} h_{1}(s) d s}{1-e^{\int_{0}^{T} h_{1}(s) d s}} \int_{-\infty}^{u} a_{1}(u, s) f_{1}(x(s), y(s)) d s d u
\end{aligned}
$$

and

$$
\begin{aligned}
& E_{2}(x, y)(t) \\
& =\frac{c_{2}(t) y\left(t-\tau_{2}(t)\right)}{1-\tau_{2}^{\prime}(t)}-\int_{t}^{t+T} \frac{e^{\int_{u}^{t+T} h_{2}(s) d s}}{1-e^{\int_{0}^{T} h_{2}(s) d s}} r_{2}(u) y\left(u-\tau_{2}(u)\right) d u \\
& \quad+\int_{t}^{t+T} \frac{e^{\int_{u}^{t+T}} h_{2}(s) d s}{1-e^{\int_{0}^{T} h_{2}(s) d s}} G_{2}\left(u, x(u), y(u), x\left(u-\tau_{1}(u)\right), y\left(u-\tau_{2}(u)\right)\right) d u \\
& \quad+\int_{t}^{t+T} \frac{e^{\int_{u}^{t+T}} h_{2}(s) d s}{1-e^{\int_{0}^{T} h_{2}(s) d s}} \int_{-\infty}^{u} a_{2}(u, s) f_{2}(x(s), y(s)) d s d u
\end{aligned}
$$

To apply Lemma 2.1, we need to construct two mappings, one is a contraction and the other is continuous and compact. Therefore, we state 
(2.11) as

$$
E_{1}(x, y)(t)=B_{1}(x, y)(t)+A_{1}(x, y)(t),
$$

where $B_{1}, A_{1}: \Omega_{x, y} \rightarrow P_{T}$ are given by

$$
B_{1}(x, y)(t)=\frac{c_{1}(t) x\left(t-\tau_{1}(t)\right)}{1-\tau_{1}^{\prime}(t)},
$$

and

$$
\begin{aligned}
A_{1}(x, y)(t) & =-\int_{t}^{t+T} \frac{e^{\int_{u}^{t+T} h_{1}(s) d s}}{1-e^{\int_{0}^{T} h_{1}(s) d s}} r_{1}(u) x\left(u-\tau_{1}(u)\right) d u \\
& +\int_{t}^{t+T} \frac{e^{\int_{u}^{t+T} h_{1}(s) d s}}{1-e^{\int_{0}^{T} h_{1}(s) d s}} G_{1}\left(u, x(u), y(u), x\left(u-\tau_{1}(u)\right), y\left(u-\tau_{2}(u)\right)\right) d u \\
& +\int_{t}^{t+T} \frac{e^{\int_{u}^{t+T}} h_{1}(s) d s}{1-e^{\int_{0}^{T} h_{1}(s) d s}} \int_{-\infty}^{u} a_{1}(u, s) f_{1}(x(s), y(s)) d s d u
\end{aligned}
$$

And we state (2.12) as

$$
E_{2}(x, y)(t)=B_{2}(x, y)(t)+A_{2}(x, y)(t)
$$

where $B_{2}, A_{2}: \Omega_{x, y} \rightarrow P_{T}$ are given by

$$
B_{2}(x, y)(t)=\frac{c_{2}(t) y\left(t-\tau_{2}(t)\right)}{1-\tau_{2}^{\prime}(t)},
$$

and

$$
\begin{aligned}
A_{2}(x, y)(t) & =-\int_{t}^{t+T} \frac{e^{\int_{u}^{t+T} h_{2}(s) d s}}{1-e^{\int_{0}^{T} h_{2}(s) d s}} r_{2}(u) y\left(u-\tau_{2}(u)\right) d u \\
& +\int_{t}^{t+T} \frac{e^{\int_{u}^{t+T} h_{2}(s) d s}}{1-e^{\int_{0}^{T} h_{2}(s) d s}} G_{2}\left(u, x(u), y(u), x\left(u-\tau_{1}(u)\right), y\left(u-\tau_{2}(u)\right)\right) d u \\
& +\int_{t}^{t+T} \frac{e^{\int_{u}^{t+T} h_{2}(s) d s}}{1-e^{\int_{0}^{T} h_{2}(s) d s}} \int_{-\infty}^{u} a_{2}(u, s) f_{2}(x(s), y(s)) d s d u
\end{aligned}
$$

Now for $(x, y) \in \Omega_{x, y}$ we can define the operators $B, A: \Omega_{x, y} \rightarrow P_{T}$ by

$$
B(x, y)(t)=\left(B_{1}(x, y)(t), B_{2}(x, y)(t)\right)
$$

and

$$
A(x, y)(t)=\left(A_{1}(x, y)(t), A_{2}(x, y)(t)\right)
$$


Observe that, since the functions $G_{i}\left(t, x_{1}, x_{2}, x_{3}, x_{4}\right), i=1,2$ is Lipschitz continuous in $x_{1}, x_{2}, x_{3}, x_{4}$ and $f_{i}\left(x_{1}, x_{2}\right), i=1,2$ is Lipschitz continuous in $x_{1}, x_{2}$ we have

$$
\begin{aligned}
\mid G_{1} & \left(t, x_{1}, x_{2}, x_{3}, x_{4}\right) \mid \\
& =\left|G_{1}\left(t, x_{1}, x_{2}, x_{3}, x_{4}\right)-G_{1}(t, 0,0,0,0)+G_{1}(t, 0,0,0,0)\right| \\
& \leq\left|G_{1}\left(t, x_{1}, x_{2}, x_{3}, x_{4}\right)-G_{1}(t, 0,0,0,0)\right|+\left|G_{1}(t, 0,0,0,0)\right| \\
& \leq \sum_{j=1}^{4} N_{j}\left|x_{j}\right| \\
\mid G_{2} & \left(t, x_{1}, x_{2}, x_{3}, x_{4}\right) \mid \\
& =\left|G_{2}\left(t, x_{1}, x_{2}, x_{3}, x_{4}\right)-G_{2}(t, 0,0,0,0)+G_{2}(t, 0,0,0,0)\right| \\
& \leq\left|G_{2}\left(t, x_{1}, x_{2}, x_{3}, x_{4}\right)-G_{2}(t, 0,0,0,0)\right|+\left|G_{2}(t, 0,0,0,0)\right| \\
\leq & \sum_{j=1}^{4} R_{j}\left|x_{j}\right|, \\
\left|f_{1}\left(x_{1}, x_{2}\right)\right| & =\left|f_{1}\left(x_{1}, x_{2}\right)-f_{1}(0,0)+f_{1}(0,0)\right| \\
\leq & \left|f_{1}\left(x_{1}, x_{2}\right)-f_{1}(0,0)\right|+\left|f_{1}(0,0)\right| \\
\leq & \sum_{j=1}^{2} d_{j}\left|x_{j}\right|,
\end{aligned}
$$

and

$$
\begin{aligned}
\left|f_{2}\left(x_{1}, x_{2}\right)\right| & =\left|f_{2}\left(x_{1}, x_{2}\right)-f_{2}(0,0)+f_{2}(0,0)\right| \\
& \leq\left|f_{2}\left(x_{1}, x_{2}\right)-f_{2}(0,0)\right|+\left|f_{2}(0,0)\right| \\
& \leq \sum_{j=1}^{2} q_{j}\left|x_{j}\right| .
\end{aligned}
$$

Theorem 2.2. Suppose (1.2), (1.3) and (2.1)-(2.9) hold. Suppose that

$$
\beta_{1}+L_{1} \sum_{j=1}^{4} N_{j}+\alpha_{1} \sum_{j=1}^{2} d_{j} \leq 1, \text { and } \beta_{2}+L_{2} \sum_{j=1}^{4} R_{j}+\alpha_{2} \sum_{j=1}^{2} q_{j} \leq 1 .
$$

Then (1.1) has a T-periodic solution.

Proof. In order to prove that (1.1) has a $T$-periodic solution, we shall make sure that A and B satisfy the conditions of Lemma 2.1. For all $(x, y) \in \Omega_{x, y}$, 
we have $(x, y)(t+T)=(x, y)(t)$ and $\|(x, y)\| \leq M$. Now let us discuss $B(x, y)+A(x, y)$. We have

$$
\begin{aligned}
B_{1}(x, y)(t+T) & =\frac{c_{1}(t+T) x\left(t+T-\tau_{1}(t+T)\right)}{1-\tau_{1}^{\prime}(t+T)} \\
& =\frac{c_{1}(t) x\left(t-\tau_{1}(t)\right)}{1-\tau_{1}^{\prime}(t)}=B_{1}(x, y)(t),
\end{aligned}
$$

and

$$
\begin{aligned}
& A_{1}(x, y)(t+T) \\
& =-\int_{t+T}^{t+2 T} \frac{e^{\int_{u}^{t+2 T} h_{1}(s) d s}}{1-e^{\int_{0}^{T}} h_{1}(s) d s} r_{1}(u) x\left(u-\tau_{1}(u)\right) d u \\
& +\int_{t+T}^{t+2 T} \frac{e^{\int_{u}^{t+2 T} h_{1}(s) d s}}{1-e^{\int_{0}^{T} h_{1}(s) d s}} G_{1}\left(u, x(u), y(u), x\left(u-\tau_{1}(u)\right), y\left(u-\tau_{2}(u)\right)\right) d u \\
& +\int_{t+T}^{t+2 T} \frac{e^{\int_{u}^{t+2 T} h_{1}(s) d s}}{1-e^{\int_{0}^{T} h_{1}(s) d s}} \int_{-\infty}^{u} a_{1}(u, s) f_{1}(x(s), y(s)) d s d u \\
& =-\int_{t}^{t+T} \frac{e^{\int_{u}^{t+T} h_{1}(s) d s}}{1-e^{\int_{0}^{T} h_{1}(s) d s}} r_{1}(u) x\left(u-\tau_{1}(u)\right) d u \\
& +\int_{t}^{t+T} \frac{e^{\int_{u}^{t+T} h_{1}(s) d s}}{1-e^{\int_{0}^{T} h_{1}(s) d s}} G_{1}\left(u, x(u), y(u), x\left(u-\tau_{1}(u)\right), y\left(u-\tau_{2}(u)\right)\right) d u \\
& +\int_{t}^{t+T} \frac{e^{e_{u}^{t+T} h_{1}(s) d s}}{1-e^{\int_{0}^{T} h_{1}(s) d s}} \int_{-\infty}^{u} a_{1}(u, s) f_{1}(x(s), y(s)) d s d u \\
& =A_{1}(x, y)(t) \text {. }
\end{aligned}
$$

Then $E_{1}(x, y)(t+T)=E_{1}(x, y)(t)$. In a similar way we can easily show that $E_{2}(x, y)(t+T)=E_{2}(x, y)(t)$. Therefore, $E(x, y)(t+T)=E(x, y)(t)$.

For any $(x, y) \in \Omega_{x, y}$, we will show that $|E(x, y)(t)| \leq M$. In view of the above estimates, we have

$$
\left|B_{1}(x, y)(t)\right| \leq\left|\frac{c_{1}(t)}{1-\tau_{1}^{\prime}(t)}\right|\left|x\left(t-\tau_{1}(t)\right)\right| \leq \mu_{1} M,
$$

and

$$
\left|A_{1}(x, y)(t)\right| \leq \int_{t}^{t+T}\left|\frac{e^{\int_{u}^{t+T} h_{1}(s) d s}}{1-e^{\int_{0}^{T} h_{1}(s) d s}}\right|\left|r_{1}(u)\right|\left|x\left(u-\tau_{1}(u)\right)\right| d u
$$




$$
\begin{aligned}
& +\int_{t}^{t+T}\left|\frac{e^{\int_{u}^{t+T} h_{1}(s) d s}}{1-e^{\int_{0}^{T} h_{1}(s) d s}}\right| \mid G_{1}\left(u, x(u), y(u), x\left(u-\tau_{1}(u)\right),\right. \\
& \left.\quad y\left(u-\tau_{2}(u)\right)\right) \mid d u \\
& +\int_{t}^{t+T}\left|\frac{e^{\int_{u}^{t+T} h_{1}(s) d s}}{1-e^{\int_{0}^{T} h_{1}(s) d s}}\right| \int_{-\infty}^{u}\left|a_{1}(u, s)\right|\left|f_{1}(x(s), y(s))\right| d s d u \\
& \leq \beta_{1} M+L_{1} K_{1}+\alpha_{1} M_{1} .
\end{aligned}
$$

As a consequence of (2.10)

$$
\frac{L_{1} K_{1}+\alpha_{1} M_{1}}{1-\mu_{1}-\beta_{1}} \leq M,
$$

so,

$$
L_{1} K_{1}+\alpha_{1} M_{1} \leq\left(1-\mu_{1}-\beta_{1}\right) M
$$

This implies that

$$
\begin{aligned}
\left|E_{1}(x, y)(t)\right| & \leq \mu_{1} M+\beta_{1} M+L_{1} K_{1}+\alpha_{1} M_{1} \\
& \leq \mu_{1} M+\beta_{1} M+\left(1-\mu_{1}-\beta_{1}\right) M=M
\end{aligned}
$$

In a similar way we can easily show that

$$
\left|E_{2}(x, y)(t)\right| \leq M
$$

Thus, $E$ maps $\Omega_{x, y}$ into itself, i.e. $E\left(\Omega_{x, y}\right) \subseteq \Omega_{x, y}$. We will now show that $A$ is continuous. Let $\left\{\left(x_{n}, y_{n}\right)\right\}$ be a sequence in $\Omega_{x, y}$ such that

$$
\lim _{n \rightarrow \infty}\left\|\left(x_{n}, y_{n}\right)-(x, y)\right\|=0
$$

Since $\Omega_{x, y}$ is closed, we have $(x, y) \in \Omega_{x, y}$. Then by the definition of $A$ we have

$$
\begin{aligned}
& \left\|A\left(x_{n}, y_{n}\right)-A(x, y)\right\| \\
& =\max \left\{\max _{t \in[0, T]}\left|A_{1}\left(x_{n}, y_{n}\right)(t)-A_{1}(x, y)(t)\right|, \max _{t \in[0, T]}\left|A_{2}\left(x_{n}, y_{n}\right)(t)-A_{2}(x, y)(t)\right|\right\},
\end{aligned}
$$

in which

$$
\left|A_{1}\left(x_{n}, y_{n}\right)(t)-A_{1}(x, y)(t)\right|
$$




$$
\begin{aligned}
\leq & \int_{t}^{t+T}\left|\frac{e^{\int_{u}^{t+T} h_{1}(s) d s}}{1-e^{\int_{0}^{T} h_{1}(s) d s} \mid}\right| r_{1}(u)|| x_{n}\left(u-\tau_{1}(u)\right)-x\left(u-\tau_{1}(u)\right) \mid d u \\
& +\int_{t}^{t+T}\left|\frac{e^{\int_{u}^{t+T} h_{1}(s) d s}}{1-e^{\int_{0}^{T} h_{1}(s) d s}}\right| \mid G_{1}\left(u, x_{n}(u), y_{n}(u), x_{n}\left(u-\tau_{1}(u)\right), y_{n}\left(u-\tau_{2}(u)\right)\right) \\
& -G_{1}\left(u, x(u), y(u), x\left(u-\tau_{1}(u)\right), y\left(u-\tau_{2}(u)\right)\right) \mid d u \\
& +\int_{t}^{t+T}\left|\frac{e^{\int_{u}^{t+T} h_{1}(s) d s}}{1-e^{\int_{0}^{T} h_{1}(s) d s}}\right| \int_{-\infty}^{u}\left|a_{1}(u, s)\right|\left|f_{1}\left(x_{n}(s), y_{n}(s)\right)-f_{1}(x(s), y(s))\right| d s d u,
\end{aligned}
$$

the continuity of $G_{1}$ and $f_{1}$ along with the Lebesgue dominated convergence theorem implies that

$$
\lim _{n \rightarrow \infty} \max _{t \in[0, T]}\left|A_{1}\left(x_{n}, y_{n}\right)(t)-A_{1}(x, y)(t)\right|=0
$$

By a similar argument we can easily argue that

$$
\lim _{n \rightarrow \infty} \max _{t \in[0, T]}\left|A_{2}\left(x_{n}, y_{n}\right)(t)-A_{2}(x, y)(t)\right|=0
$$

Thus

$$
\lim _{n \rightarrow \infty}\left\|A\left(x_{n}, y_{n}\right)-A(x, y)\right\|=0 .
$$

This result proves that $A$ is continuous.

We now have to show that $A$ is compact. For $n \in \mathbb{N}$, let $\left(x_{n}, y_{n}\right) \in \Omega_{x, y}$, we have

$$
\begin{aligned}
& \left|A_{1}\left(x_{n}, y_{n}\right)(t)\right| \\
& \leq \int_{t}^{t+T}\left|\frac{e^{\int_{u}^{t+T} h_{1}(s) d s}}{1-e^{\int_{0}^{T} h_{1}(s) d s}}\right|\left|r_{1}(u)\right|\left|x_{n}\left(u-\tau_{1}(u)\right)\right| d u \\
& \quad+\int_{t}^{t+T}\left|\frac{e^{\int_{u}^{t+T} h_{1}(s) d s}}{1-e^{\int_{0}^{T} h_{1}(s) d s}}\right|\left|G_{1}\left(u, x_{n}(u), y_{n}(u), x_{n}\left(u-\tau_{1}(u)\right), y_{n}\left(u-\tau_{2}(u)\right)\right)\right| d u \\
& \quad+\int_{t}^{t+T}\left|\frac{e^{\int_{u}^{t+T}} h_{1}(s) d s}{1-e^{\int_{0}^{T} h_{1}(s) d s}}\right| \int_{-\infty}^{u}\left|a_{1}(u, s)\right|\left|f_{1}\left(x_{n}(s), y_{n}(s)\right)\right| d s d u \\
& \leq\left(\beta_{1}+L_{1} \sum_{j=1}^{4} N_{j}+\alpha_{1} \sum_{j=1}^{2} d_{j}\right) M \leq M .
\end{aligned}
$$


In a similar way we can easily show that

$$
\left|A_{2}\left(x_{n}, y_{n}\right)(t)\right| \leq\left(\beta_{2}+L_{2} \sum_{j=1}^{4} R_{j}+\alpha_{2} \sum_{j=1}^{2} q_{j}\right) M \leq M .
$$

Thus

$$
\left\|A\left(x_{n}, y_{n}\right)\right\| \leq M
$$

If we calculate $\left(A\left(x_{n}, y_{n}\right)\right)^{\prime}(t)$, then

$$
\begin{aligned}
& \left(A_{1}\left(x_{n}, y_{n}\right)\right)^{\prime}(t) \\
& \leq G_{1}\left(t, x_{n}(t), y_{n}(t), x_{n}\left(t-\tau_{1}(t)\right), y_{n}\left(t-\tau_{2}(t)\right)\right)-r_{1}(t) x_{n}\left(t-\tau_{1}(t)\right) \\
& \quad+\int_{-\infty}^{t} a_{1}(t, s) f_{1}\left(x_{n}(s), y_{n}(s)\right) d s+h_{1}(t) \\
& \quad \times\left[\int_{t}^{t+T} \frac{e^{\int_{u}^{t+T}} h_{1}(s) d s}{1-e^{\int_{0}^{T} h_{1}(s) d s}} G_{1}\left(u, x_{n}(u), y_{n}(u), x_{n}\left(u-\tau_{1}(u)\right), y_{n}\left(u-\tau_{2}(u)\right)\right) d u\right. \\
& \quad-\int_{t}^{t+T} \frac{e^{\int_{u}^{t+T}} h_{1}(s) d s}{1-e^{\int_{0}^{T}} h_{1}(s) d s} r_{1}(u) x_{n}\left(u-\tau_{1}(u)\right) d u \\
& \left.\quad+\int_{t}^{t+T} \frac{e^{\int_{u}^{t+T} h_{1}(s) d s}}{1-e^{\int_{0}^{T} h_{1}(s) d s}} \int_{-\infty}^{u} a_{1}(u, s) f_{1}(x(s), y(s)) d s d u\right] \\
& =G_{1}\left(t, x_{n}(t), y_{n}(t), x_{n}\left(t-\tau_{1}(t)\right), y_{n}\left(t-\tau_{2}(t)\right)\right)-r_{1}(t) x_{n}\left(t-\tau_{1}(t)\right) \\
& \quad+\int_{-\infty}^{t} a_{1}(t, s) f_{1}\left(x_{n}(s), y_{n}(s)\right) d s+h_{1}(t) A_{1}\left(x_{n}, y_{n}\right)(t) .
\end{aligned}
$$

Hence, for some positive constant $D_{1}$, we obtain

$$
\begin{aligned}
\mid & \left(A_{1}\left(x_{n}, y_{n}\right)\right)^{\prime}(t) \mid \\
= & \left|G_{1}\left(t, x_{n}(t), y_{n}(t), x_{n}\left(t-\tau_{1}(t)\right), y_{n}\left(t-\tau_{2}(t)\right)\right)\right|+\left|r_{1}(t)\right|\left|x_{n}\left(t-\tau_{1}(t)\right)\right| \\
& \quad+\int_{-\infty}^{t}\left|a_{1}(t, s)\right|\left|f_{1}\left(x_{n}(s), y_{n}(s)\right)\right| d s+\left|h_{1}(t)\right|\left|A_{1}\left(x_{n}, y_{n}\right)(t)\right| \\
\leq & {\left[\sum_{j=1}^{4} N_{j}+\theta_{1} \sum_{j=1}^{2} d_{j}+\theta_{3}+\theta_{4}\right] M \leq D_{1}, }
\end{aligned}
$$

where $\sup _{t \in[0, T]}\left|r_{1}(t)\right|=\theta_{3}, \sup _{t \in[0, T]}\left|h_{1}(t)\right|=\theta_{4}$. In a similar way we can 
show for some positive constant $D_{2}$ that

$$
\left|\left(A_{2}\left(x_{n}, y_{n}\right)\right)^{\prime}(t)\right| \leq\left[\sum_{j=1}^{4} R_{j}+\theta_{2} \sum_{j=1}^{2} q_{j}+\theta_{5}+\theta_{6}\right] M \leq D_{2},
$$

where $\sup _{t \in[0, T]}\left|r_{2}(t)\right|=\theta_{5}, \sup _{t \in[0, T]}\left|h_{2}(t)\right|=\theta_{6}$. Thus

$$
\left\|\left(A\left(x_{n}, y_{n}\right)\right)^{\prime}\right\| \leq D
$$

where $D=\max \left(D_{1}, D_{2}\right)$. Thus, the sequence $\left(A\left(x_{n}, y_{n}\right)\right)$ is uniformly bounded and equi-continuous. The Arzela-Ascoli theorem implies that there exists a subsequence $\left(A\left(x_{n_{k}}, y_{n_{k}}\right)\right)$ of $\left(A\left(x_{n}, y_{n}\right)\right)$ converges uniformly to a continuous $T$-periodic function $\left(x^{*}, y^{*}\right)$. Thus, $A$ is compact.

For all $\left(x_{1}, y_{1}\right),\left(x_{2}, y_{2}\right) \in \Omega_{x, y}$

$$
\begin{aligned}
\left|B_{1}\left(x_{1}, y_{1}\right)(t)-B_{1}\left(x_{2}, y_{2}\right)(t)\right| & =\left|\frac{c_{1}(t) x_{1}\left(t-\tau_{1}(t)\right)}{1-\tau_{1}^{\prime}(t)}-\frac{c_{1}(t) x_{2}\left(t-\tau_{1}(t)\right)}{1-\tau_{1}^{\prime}(t)}\right| \\
& =\left|\frac{c_{1}(t)}{1-\tau_{1}^{\prime}(t)}\right|\left|x_{1}\left(t-\tau_{1}(t)\right)-x_{2}\left(t-\tau_{1}(t)\right)\right| \\
& \leq \mu_{1}\left|x_{1}\left(t-\tau_{1}(t)\right)-x_{2}\left(t-\tau_{1}(t)\right)\right|
\end{aligned}
$$

hence $B_{1}$ is contraction because $\mu_{1}<1$. In a similar way we can easily show that

$$
\left|B_{2}\left(x_{1}, y_{1}\right)(t)-B_{2}\left(x_{2}, y_{2}\right)(t)\right| \leq \mu_{2}\left|y_{1}\left(t-\tau_{2}(t)\right)-y_{2}\left(t-\tau_{2}(t)\right)\right|
$$

hence $B_{2}$ is contraction because $\mu_{2}<1$. Then

$$
\begin{aligned}
& \left|B\left(x_{1}, y_{1}\right)(t)-B\left(x_{2}, y_{2}\right)(t)\right| \\
& \quad=\max \left\{\left|B_{1}\left(x_{1}, y_{1}\right)(t)-B_{1}\left(x_{2}, y_{2}\right)(t)\right|,\left|B_{2}\left(x_{1}, y_{1}\right)(t)-B_{2}\left(x_{2}, y_{2}\right)(t)\right|\right\}
\end{aligned}
$$

this implies that

$$
\left\|B\left(x_{1}, y_{1}\right)-B\left(x_{2}, y_{2}\right)\right\| \leq \mu\left\|\left(x_{1}, y_{1}\right),\left(x_{2}, y_{2}\right)\right\|
$$

Hence $B$ is contraction.

Thus, the conditions of Lemma 2.1 are satisfied and there is a $(x, y) \in$ $\Omega_{x, y}$, such that $(x, y)=A(x, y)+B(x, y)$. 
In the next theorem we relax condition (2.2).

Theorem 2.3. Suppose (1.2), (1.3), (2.1) and (2.3)-(2.9) hold. Suppose that

$$
\beta_{1}+L_{1} \sum_{j=1}^{4} N_{j}+\alpha_{1} \sum_{j=1}^{2} d_{j} \leq 1, \text { and } \beta_{2}+L_{2} \sum_{j=1}^{4} R_{j}+\alpha_{2} \sum_{j=1}^{2} q_{j} \leq 1 .
$$

In addition, we assume the existence of continuous nondecreasing function $W_{2}$ such that

$$
\left|f_{2}(x, y)\right| \leq f_{2}(|x|, y) \leq Q_{2} W_{2}(|x|)
$$

for some positive constant $Q_{2}$, and for $u>0$ we ask that

$$
\frac{W_{2}(u)}{u} \leq \frac{1-\mu_{2}-\beta_{2}-\frac{L_{2} K_{2}}{M}}{\alpha_{2} Q_{2}}
$$

Then (1.1) has a T-periodic solution.

Proof. Set

$$
M=\max \left\{\frac{L_{1} K_{1}+\alpha_{1} M_{1}}{1-\mu_{1}-\beta_{1}}, \frac{L_{2} K_{2}+\alpha_{2} Q_{2} W_{2}(M)}{1-\mu_{2}-\beta_{2}}\right\} .
$$

Note that due to (2.20) we have

$$
M \geq \frac{L_{2} K_{2}+\alpha_{2} Q_{2} W_{2}(M)}{1-\mu_{2}-\beta_{2}},
$$

and hence (2.20) is well defined. For any $(x, y) \in \Omega_{x, y}$, we have by the proof of the previous theorem that

$$
\left|E_{1}(x, y)(t)\right| \leq M
$$

Thus

$$
\left|B_{2}(x, y)(t)\right| \leq\left|\frac{c_{2}(t)}{1-\tau_{2}^{\prime}(t)}\right|\left|y\left(t-\tau_{2}(t)\right)\right| \leq \mu_{2} M
$$

and

$$
\left|A_{2}(x, y)(t)\right|
$$




$$
\begin{aligned}
\leq & \int_{t}^{t+T}\left|\frac{e^{\int_{u}^{t+T} h_{2}(s) d s}}{1-e^{\int_{0}^{T} h_{2}(s) d s}}\right|\left|r_{2}(u)\right|\left|x\left(u-\tau_{1}(u)\right)\right| d u \\
& +\int_{t}^{t+T}\left|\frac{e^{\int_{u}^{t+T} h_{2}(s) d s}}{1-e^{\int_{0}^{T} h_{2}(s) d s}}\right|\left|G_{2}\left(u, x(u), y(u), x\left(u-\tau_{1}(u)\right), y\left(u-\tau_{2}(u)\right)\right)\right| d u \\
& +\int_{t}^{t+T}\left|\frac{e^{\int_{u}^{t+T} h_{2}(s) d s}}{1-e^{\int_{0}^{T} h_{2}(s) d s}}\right| \int_{-\infty}^{t}\left|a_{2}(u, s)\right| f_{2}(|x(s)|, y(s)) d s d u \\
\leq & M \int_{t}^{t+T}\left|\frac{e^{\int_{u}^{t+T} h_{2}(s) d s}}{1-e^{\int_{0}^{T} h_{2}(s) d s}}\right|\left|r_{2}(u)\right| d u+K_{2} \int_{t}^{t+T} \mid \frac{e^{\int_{u}^{t+T} h_{2}(s) d s}}{1-e^{\int_{0}^{T} h_{2}(s) d s} \mid d u} \\
& +Q_{2} W_{2}(M) \int_{t}^{t+T}\left|\frac{e^{\int_{u}^{t+T}} h_{2}(s) d s}{1-e^{\int_{0}^{T} h_{2}(s) d s}}\right| \int_{-\infty}^{t}\left|a_{2}(u, s)\right| d s d u \\
\leq & \beta_{2} M+L_{2} K_{2}+\alpha_{2} Q_{2} W_{2}(M) .
\end{aligned}
$$

As a consequence of (2.21)

$$
\frac{L_{2} K_{2}+\alpha_{2} Q_{2} W_{2}(M)}{1-\mu_{2}-\beta_{2}} \leq M,
$$

so,

$$
L_{2} K_{2}+\alpha_{2} Q_{2} W_{2}(M) \leq\left(1-\mu_{2}-\beta_{2}\right) M
$$

This implies that

$$
\begin{aligned}
\left|E_{2}(x, y)(t)\right| & \leq \mu_{2} M+\beta_{2} M+L_{2} K_{2}+\alpha_{2} Q_{2} W_{2}(M) \\
& \leq \mu_{2} M+\beta_{2} M+\left(1-\mu_{2}-\beta_{2}\right) M=M .
\end{aligned}
$$

The rest of the proof follows along the lines of the proof of Theorem 2.2 .

In the next theorem we relax condition (2.1).

Theorem 2.4. Suppose (1.2), (1.3), (2.2) and (2.3)-(2.9) hold. Suppose that

$$
\beta_{1}+L_{1} \sum_{j=1}^{4} N_{j}+\alpha_{1} \sum_{j=1}^{2} d_{j} \leq 1 \text { and } \beta_{2}+L_{2} \sum_{j=1}^{4} R_{j}+\alpha_{2} \sum_{j=1}^{2} q_{j} \leq 1 .
$$

In addition, we assume the existence of continuous nondecreasing function 
$W_{1}$ such that

$$
\left|f_{1}(x, y)\right| \leq f_{1}(x,|y|) \leq Q_{1} W_{1}(|y|)
$$

for some positive constant $Q_{1}$, and for $u>0$ we ask that

$$
\frac{W_{1}(u)}{u} \leq \frac{1-\mu_{1}-\beta_{1}-\frac{L_{1} K_{1}}{M}}{\alpha_{1} Q_{1}} .
$$

Then (1.1) has a T-periodic solution.

Proof. Set

$$
M=\max \left\{\frac{L_{1} K_{1}+\alpha_{1} Q_{1} W_{1}(M)}{1-\mu_{1}-\beta_{1}}, \frac{L_{2} K_{2}+\alpha_{2} M_{2}}{1-\mu_{1}-\beta_{1}}\right\} .
$$

Note that due to (2.23) we have

$$
M \geq \frac{L_{1} K_{1}+\alpha_{1} Q_{1} W_{1}(M)}{1-\mu_{1}-\beta_{1}},
$$

and hence (2.23) is well defined. For any $(x, y) \in \Omega_{x, y}$, we have by the proof of the previous theorem that

$$
\left|E_{2}(x, y)(t)\right| \leq M
$$

Thus

$$
\left|B_{1}(x, y)(t)\right| \leq\left|\frac{c_{1}(t)}{1-\tau_{1}^{\prime}(t)}\right|\left|x\left(t-\tau_{1}(t)\right)\right| \leq \mu_{1} M
$$

and

$$
\begin{aligned}
& \left|A_{1}(x, y)(t)\right| \\
& \leq \int_{t}^{t+T}\left|\frac{e^{\int_{u}^{t+T} h_{1}(s) d s}}{1-e^{\int_{0}^{T} h_{1}(s) d s}}\right|\left|r_{1}(u)\right|\left|x\left(u-\tau_{1}(u)\right)\right| d u \\
& \quad+\int_{t}^{t+T}\left|\frac{e^{\int_{u}^{t+T} h_{1}(s) d s}}{1-e^{\int_{0}^{T} h_{1}(s) d s}}\right|\left|G_{1}\left(u, x(u), y(u), x\left(u-\tau_{1}(u)\right), y\left(u-\tau_{2}(u)\right)\right)\right| d u \\
& \quad+\int_{t}^{t+T}\left|\frac{e^{\int_{u}^{t+T}} h_{1}(s) d s}{1-e^{\int_{0}^{T} h_{1}(s) d s}}\right| \int_{-\infty}^{t}\left|a_{1}(u, s)\right| f_{1}(x(s),|y(s)|) d s d u
\end{aligned}
$$




$$
\begin{aligned}
\leq & M \int_{t}^{t+T}\left|\frac{e^{\int_{u}^{t+T} h_{1}(s) d s}}{1-e^{\int_{0}^{T} h_{1}(s) d s}}\right|\left|r_{1}(u)\right| d u+K_{1} \int_{t}^{t+T}\left|\frac{e^{\int_{u}^{t+T} h_{1}(s) d s}}{1-e^{\int_{0}^{T} h_{1}(s) d s}}\right| d u \\
& +Q_{1} W_{1}(M) \int_{t}^{t+T}\left|\frac{e^{\int_{u}^{t+T} h_{1}(s) d s}}{1-e^{\int_{0}^{T} h_{1}(s) d s}}\right| \int_{-\infty}^{t}\left|a_{1}(u, s)\right| d s d u \\
\leq & \beta_{1} M+L_{1} K_{1}+\alpha_{1} Q_{1} W_{1}(M) .
\end{aligned}
$$

As a consequence of (2.24)

$$
\frac{L_{1} K_{1}+\alpha_{1} Q_{1} W_{1}(M)}{1-\mu_{1}-\beta_{1}} \leq M
$$

so,

$$
L_{1} K_{1}+\alpha_{1} Q_{1} W_{1}(M) \leq\left(1-\mu_{1}-\beta_{1}\right) M
$$

This implies that

$$
\begin{aligned}
\left|E_{1}(x, y)(t)\right| & \leq \mu_{1} M+\beta_{1} M+L_{1} K_{1}+\alpha_{1} Q_{1} W_{1}(M) \\
& \leq \mu_{1} M+\beta_{1} M+\left(1-\mu_{1}-\beta_{1}\right) M=M .
\end{aligned}
$$

The rest of the proof follows along the lines of the proof of Theorem 2.2 .

\section{Asymptotic Stability of Periodic Solutions}

In this section, we show that under mild conditions one obtains asymptotically periodic solutions. We do not assume the periodicity condition on the functions $a_{1}, a_{2}, c_{1}, c_{2}, \tau_{1}, \tau_{2}, G_{1}$ and $G_{2}$, we only assume $h_{1}$ and $h_{2}$ are $T$-periodic, and

$$
\int_{0}^{T} h_{1}(s) d s=0, \int_{0}^{T} h_{2}(s) d s=0 .
$$

Since $h_{1}$ and $h_{2}$ are $T$-periodic, there are constants $m_{k}$ and $M_{k}^{*}, k=1,2$ such that

$$
m_{1} \leq e^{\int_{0}^{t} h_{1}(s) d s} \leq M_{1}^{*}, m_{2} \leq e^{\int_{0}^{t} h_{1}(s) d s} \leq M_{2}^{*}
$$

Furthermore, we assume that there are positive numbers $V_{1}$ and $V_{2}$ such that

$$
\int_{t}^{\infty} \int_{-\infty}^{u}\left|a_{1}(u, s)\right| d s d u \leq V_{1}, \int_{t}^{\infty} \int_{-\infty}^{u}\left|a_{2}(u, s)\right| d s d u \leq V_{2} .
$$


In addition, we suppose that

$$
\begin{aligned}
& \sup _{t \in \mathbb{R}}\left|\frac{c_{1}(t)}{1-\tau_{1}^{\prime}(t)}\right|=\mu_{1}^{*}, \sup _{t \in \mathbb{R}}\left|\frac{c_{2}(t)}{1-\tau_{2}^{\prime}(t)}\right|=\mu_{2}^{*}, \max \left\{\mu_{1}^{*}, \mu_{2}^{*}\right\}=\mu^{*}<1, \\
& \lim _{t \rightarrow \infty} \frac{c_{1}(t)}{1-\tau_{1}^{\prime}(t)}=0, \lim _{t \rightarrow \infty} \frac{c_{2}(t)}{1-\tau_{2}^{\prime}(t)}=0 \\
& \lim _{t \rightarrow \infty} \int_{t}^{\infty} \int_{-\infty}^{u}\left|a_{1}(u, s)\right| d s d u=0, \lim _{t \rightarrow \infty} \int_{t}^{\infty} \int_{-\infty}^{u}\left|a_{2}(u, s)\right| d s d u=0 \\
& \lim _{t \rightarrow \infty} \int_{t}^{\infty} \frac{e^{\int_{0}^{t} h_{1}(s) d s}}{e^{\int_{0}^{u} h_{1}(s) d s}} d u=0, \lim _{t \rightarrow \infty} \int_{t}^{\infty} \frac{e^{\int_{0}^{t} h_{2}(s) d s}}{e^{\int_{0}^{u} h_{2}(s) d s}} d u=0 \\
& \int_{t}^{\infty}\left|r_{1}(u)\right| d u \rightarrow 0, \int_{t}^{\infty}\left|r_{2}(u)\right| d u \rightarrow 0 \text { as } t \rightarrow \infty
\end{aligned}
$$

and for positive constants $M_{k}^{*}, k=\overline{3,6}$ we ask that

$$
\int_{t}^{\infty}\left|r_{1}(u)\right| d u \leq M_{3}^{*}, \quad \int_{t}^{\infty}\left|r_{2}(u)\right| d u \leq M_{4}^{*},
$$

and

$$
\int_{t}^{\infty} \frac{e^{\int_{0}^{t} h_{1}(s) d s}}{e^{\int_{0}^{u} h_{1}(s) d s}} d u \leq M_{5}^{*}, \int_{t}^{\infty} \frac{e^{\int_{0}^{t} h_{2}(s) d s}}{e^{\int_{0}^{u} h_{2}(s) d s}} d u \leq M_{6}^{*} .
$$

Finally, we make the assumption that

$$
1-\mu_{1}^{*}-M_{1}^{*} M_{3}^{*} m_{1}^{-1}>0
$$

and

$$
1-\mu_{2}^{*}-M_{2}^{*} M_{4}^{*} m_{2}^{-1}>0
$$

Theorem 3.1. Assume (1.2), (1.3). Then $x$ and $y$ is a solution of (1.1) if and only if

$$
\begin{aligned}
x(t)= & \rho_{1} e^{\int_{0}^{t} h_{1}(s) d s}+\frac{c_{1}(t) x\left(t-\tau_{1}(t)\right)}{1-\tau_{1}^{\prime}(t)}+\int_{t}^{\infty} \frac{e^{\int_{0}^{t} h_{1}(s) d s}}{e^{\int_{0}^{u} h_{1}(s) d s}} r_{1}(u) x\left(u-\tau_{1}(u)\right) d u \\
& -\int_{t}^{\infty} \frac{e^{\int_{0}^{t} h_{1}(s) d s}}{e^{\int_{0}^{u} h_{1}(s) d s}} G_{1}\left(u, x(u), y(u), x\left(u-\tau_{1}(u)\right), y\left(u-\tau_{2}(u)\right)\right) d u \\
& -\int_{t}^{\infty} \frac{e^{\int_{0}^{t} h_{1}(s) d s}}{e^{\int_{0}^{u} h_{1}(s) d s}} \int_{-\infty}^{u} a_{1}(u, s) f_{1}(x(s), y(s)) d s d u,
\end{aligned}
$$


and

$$
\begin{aligned}
y(t)= & \rho_{2} e^{\int_{0}^{t} h_{2}(s) d s}+\frac{c_{2}(t) y\left(t-\tau_{2}(t)\right)}{1-\tau_{2}^{\prime}(t)}+\int_{t}^{\infty} \frac{e^{\int_{0}^{t} h_{2}(s) d s}}{e^{\int_{0}^{u} h_{2}(s) d s}} r_{2}(u) y\left(u-\tau_{2}(u)\right) d u \\
& -\int_{t}^{\infty} \frac{e^{\int_{0}^{t} h_{2}(s) d s}}{e^{\int_{0}^{u} h_{2}(s) d s}} G_{2}\left(u, x(u), y(u), x\left(u-\tau_{1}(u)\right), y\left(u-\tau_{2}(u)\right)\right) d u \\
& -\int_{t}^{\infty} \frac{e^{\int_{0}^{t} h_{2}(s) d s}}{e^{\int_{0}^{u} h_{2}(s) d s}} \int_{-\infty}^{u} a_{2}(u, s) f_{2}(x(s), y(s)) d s d u
\end{aligned}
$$

where $r_{1}$ and $r_{2}$ are given by (1.6) and (1.7).

Proof. Let $(x, y)$ be a solution of (1.1). Next we multiply both sides of the first equation in (1.1) by $e^{-\int_{0}^{t} h_{1}(s) d s}$, and then integrate from $t$ to $\infty$, to obtain

$$
\begin{aligned}
\int_{t}^{\infty} & {\left[x(u) e^{-\int_{0}^{u} h_{1}(s) d s}\right]^{\prime} d u } \\
= & \int_{t}^{\infty} e^{-\int_{0}^{u} h_{1}(s) d s} G_{1}\left(u, x(u), y(u), x\left(u-\tau_{1}(u)\right), y\left(u-\tau_{2}(u)\right)\right) d u \\
& +\int_{t}^{\infty} e^{-\int_{0}^{u} h_{1}(s) d s} c_{1}(u) x^{\prime}\left(u-\tau_{1}(u)\right) d u \\
& +\int_{t}^{\infty} e^{-\int_{0}^{u} h_{1}(s) d s} \int_{-\infty}^{u} a_{1}(u, s) f_{1}(x(s), y(s)) d s d u
\end{aligned}
$$

Consequently, we have

$$
\begin{aligned}
\rho_{1}^{*}- & x(t) e^{-\int_{0}^{t} h_{1}(s) d s} \\
= & \int_{t}^{\infty} e^{-\int_{0}^{u} h_{1}(s) d s} G_{1}\left(u, x(u), y(u), x\left(u-\tau_{1}(u)\right), y\left(u-\tau_{2}(u)\right)\right) d u \\
& +\int_{t}^{\infty} e^{-\int_{0}^{u} h_{1}(s) d s} c_{1}(u) x^{\prime}\left(u-\tau_{1}(u)\right) d u \\
& +\int_{t}^{\infty} e^{-\int_{0}^{u} h_{1}(s) d s} \int_{-\infty}^{u} a_{1}(u, s) f_{1}(x(s), y(s)) d s d u .
\end{aligned}
$$

Multiply both sides with $e^{\int_{0}^{t} h_{1}(s) d s}$, we obtain

$$
\begin{aligned}
x(t)= & \rho_{1}^{*} e^{\int_{0}^{t} h_{1}(s) d s} \\
& -\int_{t}^{\infty} \frac{e^{\int_{0}^{t} h_{1}(s) d s}}{e^{\int_{0}^{u} h_{1}(s) d s}} G_{1}\left(u, x(u), y(u), x\left(u-\tau_{1}(u)\right), y\left(u-\tau_{2}(u)\right)\right) d u
\end{aligned}
$$




$$
\begin{aligned}
& -\int_{t}^{\infty} \frac{e^{\int_{0}^{t} h_{1}(s) d s}}{e^{\int_{0}^{u} h_{1}(s) d s}} c_{1}(u) x^{\prime}\left(u-\tau_{1}(u)\right) d u \\
& -\int_{t}^{\infty} \frac{e^{\int_{0}^{t} h_{1}(s) d s}}{e^{\int_{0}^{u} h_{1}(s) d s}} \int_{-\infty}^{u} a_{1}(u, s) f_{1}(x(s), y(s)) d s d u .
\end{aligned}
$$

Letting

$$
\begin{aligned}
& \int_{t}^{\infty} \frac{e^{\int_{0}^{t} h_{1}(s) d s}}{e^{\int_{0}^{u} h_{1}(s) d s}} c_{1}(u) x^{\prime}\left(u-\tau_{1}(u)\right) d u \\
& \quad=\int_{t}^{\infty} \frac{e^{\int_{0}^{t} h_{1}(s) d s}}{e^{\int_{0}^{u} h_{1}(s) d s}} \frac{c_{1}(u)}{1-\tau_{1}^{\prime}(u)}\left(1-\tau_{1}^{\prime}(u)\right) x^{\prime}\left(u-\tau_{1}(u)\right) d u .
\end{aligned}
$$

Performing an integration by parts, we get

$$
\begin{aligned}
& \int_{t}^{\infty} \frac{e^{\int_{0}^{t} h_{1}(s) d s}}{e^{\int_{0}^{u} h_{1}(s) d s}} c_{1}(u) x^{\prime}\left(u-\tau_{1}(u)\right) d u \\
& =\left[\frac{e^{\int_{0}^{t} h_{1}(s) d s}}{e^{\int_{0}^{u} h_{1}(s) d s}} \frac{c_{1}(u) x\left(u-\tau_{1}(u)\right)}{1-\tau_{1}^{\prime}(u)}\right]_{t}^{\infty}-\int_{t}^{\infty} \frac{e^{\int_{0}^{t} h_{1}(s) d s}}{e^{\int_{0}^{u} h_{1}(s) d s}} r_{1}(u) x\left(u-\tau_{1}(u)\right) d u \\
& =\rho_{1}^{* *} e^{\int_{0}^{t} h_{1}(s) d s}-\frac{c_{1}(t) x\left(t-\tau_{1}(t)\right)}{1-\tau_{1}^{\prime}(t)}-\int_{t}^{\infty} \frac{e^{\int_{0}^{t} h_{1}(s) d s}}{e^{\int_{0}^{u} h_{1}(s) d s}} r_{1}(u) x\left(u-\tau_{1}(u)\right) d u,
\end{aligned}
$$

where $r_{1}$ is given by (1.6). Substituting (3.15) into (3.14), we obtain

$$
\begin{aligned}
x(t)= & \rho_{1} e^{\int_{0}^{t} h_{1}(s) d s}+\frac{c_{1}(t) x\left(t-\tau_{1}(t)\right)}{1-\tau_{1}^{\prime}(t)}+\int_{t}^{\infty} \frac{e^{\int_{0}^{t} h_{1}(s) d s}}{e^{\int_{0}^{u} h_{1}(s) d s}} r_{1}(u) x\left(u-\tau_{1}(u)\right) d u \\
& -\int_{t}^{\infty} \frac{e^{\int_{0}^{t} h_{1}(s) d s}}{e^{\int_{0}^{u} h_{1}(s) d s}} G_{1}\left(u, x(u), y(u), x\left(u-\tau_{1}(u)\right), y\left(u-\tau_{2}(u)\right)\right) d u \\
& -\int_{t}^{\infty} \frac{e^{\int_{0}^{t} h_{1}(s) d s}}{e^{\int_{0}^{u} h_{1}(s) d s}} \int_{-\infty}^{u} a_{1}(u, s) f_{1}(x(s), y(s)) d s d u
\end{aligned}
$$

where $\rho_{1}=\rho_{1}^{*}-\rho_{1}^{* *}$. The proof of (3.13) is similar and hence we omit it.

Theorem 3.2. Suppose that (2.1), (2.2) and (3.1)-(3.11) hold. Then system (1.1) has asymptotically T-periodic solution $(x, y)$ satisfying

$$
x(t)=x_{1}(t)+x_{2}(t), y(t)=y_{1}(t)+y_{2}(t),
$$


where

$$
x_{1}(t)=\rho_{1} e^{\int_{0}^{t} h_{1}(s) d s}, y_{1}(t)=\rho_{2} e^{\int_{0}^{t} h_{2}(s) d s}, t \in \mathbb{R},
$$

for arbitrary fixed nonzero constants $\rho_{1}, \rho_{2}$ and

$$
\lim _{t \rightarrow \infty} x_{2}(t)=\lim _{t \rightarrow \infty} y_{2}(t)=0
$$

\section{Proof. Define}

$$
\begin{aligned}
P_{T}^{*}= & \left\{(\varphi, \psi): \varphi=\varphi_{1}+\varphi_{2}, \psi=\psi_{1}+\psi_{2},\left(\varphi_{1}, \psi_{1}\right)(t+T)=\left(\varphi_{1}, \psi_{1}\right)(t),\right. \\
& \text { and } \left.\left(\varphi_{2}, \psi_{2}\right)(t) \rightarrow(0,0) \text { as } t \rightarrow \infty\right\}
\end{aligned}
$$

where both $\varphi$ and $\psi$ are real valued bounded continuous functions on $\mathbb{R}$. Then $P_{T}^{*}$ is a Banach space when endowed with the maximum norm

$$
\|(x, y)\|=\max \left\{\sup _{t \in \mathbb{R}}|x(t)|, \sup _{t \in \mathbb{R}}|y(t)|\right\} .
$$

We define a subset $\Omega_{x, y}^{*}$ of $P_{T}^{*}$ as follows. For a constant $V^{*}$ to be defined later in the proof, let

$$
\Omega_{x, y}^{*}=\left\{(x, y) \in P_{T}^{*} \text { with }\|(x, y)\| \leq V^{*}\right\}
$$

Then $\Omega_{x, y}^{*}$ is a bounded, closed and convex subset of $P_{T}^{*}$. Now for $(x, y) \in$ $\Omega_{x, y}^{*}$ we can define an operator $F: \Omega_{x, y}^{*} \rightarrow P_{T}^{*}$ by

$$
F(x, y)(t)=\left(F_{1}(x, y)(t), F_{2}(x, y)(t)\right)
$$

where

$$
\begin{aligned}
F_{1}(x, y)(t)= & \rho_{1} e^{\int_{0}^{t} h_{1}(s) d s}+\frac{c_{1}(t) x\left(t-\tau_{1}(t)\right)}{1-\tau_{1}^{\prime}(t)} \\
& +\int_{t}^{\infty} \frac{e^{\int_{0}^{t} h_{1}(s) d s}}{e^{\int_{0}^{u} h_{1}(s) d s}} r_{1}(u) x\left(u-\tau_{1}(u)\right) d u \\
& -\int_{t}^{\infty} \frac{e^{\int_{0}^{t} h_{1}(s) d s}}{e^{\int_{0}^{u} h_{1}(s) d s}} G_{1}\left(u, x(u), y(u), x\left(u-\tau_{1}(u)\right), y\left(u-\tau_{2}(u)\right)\right) d u \\
& -\int_{t}^{\infty} \frac{e^{\int_{0}^{t} h_{1}(s) d s}}{e^{\int_{0}^{u} h_{1}(s) d s}} \int_{-\infty}^{u} a_{1}(u, s) f_{1}(x(s), y(s)) d s d u,
\end{aligned}
$$


and

$$
\begin{aligned}
F_{2}(x, y)(t)= & \rho_{2} e^{\int_{0}^{t} h_{2}(s) d s}+\frac{c_{2}(t) y\left(t-\tau_{2}(t)\right)}{1-\tau_{2}^{\prime}(t)} \\
& +\int_{t}^{\infty} \frac{e^{\int_{0}^{t} h_{2}(s) d s}}{e^{\int_{0}^{u} h_{2}(s) d s}} r_{2}(u) y\left(u-\tau_{2}(u)\right) d u \\
& -\int_{t}^{\infty} \frac{e^{\int_{0}^{t} h_{2}(s) d s}}{e^{\int_{0}^{u} h_{2}(s) d s}} G_{2}\left(u, x(u), y(u), x\left(u-\tau_{1}(u)\right), y\left(u-\tau_{2}(u)\right)\right) d u \\
& -\int_{t}^{\infty} \frac{e^{\int_{0}^{t} h_{2}(s) d s}}{e^{\int_{0}^{u} h_{2}(s) d s}} \int_{-\infty}^{u} a_{2}(u, s) f_{2}(x(s), y(s)) d s d u .
\end{aligned}
$$

We will show that the mapping $F$ has a fixed point in $\Omega_{x, y}^{*}$. To apply Lemma 2.1. we need to construct two mappings, one is a contraction and the other is continuous compact. Therefore, we state (3.12) as

$$
F_{1}(x, y)(t)=B_{3}(x, y)(t)+A_{3}(x, y)(t),
$$

where $B_{3}, A_{3}: \Omega_{x, y}^{*} \rightarrow P_{T}^{*}$ are given by

$$
B_{3}(x, y)(t)=\rho_{1} e^{\int_{0}^{t} h_{1}(s) d s}+\frac{c_{1}(t) x\left(t-\tau_{1}(t)\right)}{1-\tau_{1}^{\prime}(t)},
$$

and

$$
\begin{aligned}
A_{3}(x, y)(t) & =\int_{t}^{\infty} \frac{e^{\int_{0}^{t} h_{1}(s) d s}}{e^{\int_{0}^{u}} h_{1}(s) d s} r_{1}(u) x\left(u-\tau_{1}(u)\right) d u \\
& -\int_{t}^{\infty} \frac{e^{\int_{0}^{t} h_{1}(s) d s}}{e^{\int_{0}^{u} h_{1}(s) d s}} G_{1}\left(u, x(u), y(u), x\left(u-\tau_{1}(u)\right), y\left(u-\tau_{2}(u)\right)\right) d u \\
& -\int_{t}^{\infty} \frac{e^{\int_{0}^{t} h_{1}(s) d s}}{e^{\int_{0}^{u} h_{1}(s) d s}} \int_{-\infty}^{u} a_{1}(u, s) f_{1}(x(s), y(s)) d s d u
\end{aligned}
$$

And we state (3.13) as

$$
F_{2}(x, y)(t)=B_{4}(x, y)(t)+A_{4}(x, y)(t),
$$

where $B_{4}, A_{4}: \Omega_{x, y}^{*} \rightarrow P_{T}^{*}$ are given by

$$
B_{4}(x, y)(t)=\rho_{2} e^{\int_{0}^{t} h_{2}(s) d s}+\frac{c_{2}(t) y\left(t-\tau_{2}(t)\right)}{1-\tau_{2}^{\prime}(t)},
$$


and

$$
\begin{aligned}
A_{4}(x, y)(t)= & \int_{t}^{\infty} \frac{e^{\int_{0}^{t} h_{2}(s) d s}}{e^{\int_{0}^{u} h_{2}(s) d s}} r_{2}(u) y\left(u-\tau_{2}(u)\right) d u \\
& -\int_{t}^{\infty} \frac{e^{\int_{0}^{t} h_{2}(s) d s}}{e^{\int_{0}^{u} h_{2}(s) d s}} G_{2}\left(u, x(u), y(u), x\left(u-\tau_{1}(u)\right), y\left(u-\tau_{2}(u)\right)\right) d u \\
& -\int_{t}^{\infty} \frac{e^{\int_{0}^{t} h_{2}(s) d s}}{e^{\int_{0}^{u} h_{2}(s) d s}} \int_{-\infty}^{u} a_{2}(u, s) f_{2}(x(s), y(s)) d s d u .
\end{aligned}
$$

Now for $(x, y) \in \Omega_{x, y}^{*}$ we can define the operators $B^{*}, A^{*}: \Omega_{x, y}^{*} \rightarrow P_{T}^{*}$ by

$$
\begin{aligned}
B^{*}(x, y)(t) & =\left(B_{3}(x, y)(t), B_{4}(x, y)(t)\right), \\
A^{*}(x, y)(t) & =\left(A_{3}(x, y)(t), A_{4}(x, y)(t)\right) .
\end{aligned}
$$

Set $V^{*}=\max \left\{V_{1}^{*}, V_{2}^{*}\right\}$, where

$$
\begin{aligned}
& V_{1}^{*}=\frac{K_{1} M_{5}^{*} m_{1}^{-1}+M_{1}^{*} M_{1} V_{1} m_{1}^{-1}+\rho_{1} M_{1}^{*}}{1-\mu_{1}^{*}-M_{1}^{*} M_{3}^{*} m_{1}^{-1}}, \\
& V_{2}^{*}=\frac{K_{2} M_{6}^{*} m_{2}^{-1}+M_{2}^{*} M_{2} V_{2} m_{1}^{-1}+\rho_{2} M_{2}^{*}}{1-\mu_{2}^{*}-M_{2}^{*} M_{4}^{*} m_{2}^{-1}} .
\end{aligned}
$$

We note that $V^{*}$ is well defined due to (3.10) and (3.11). First, we demonstrate that $F\left(\Omega_{x, y}^{*}\right) \subseteq \Omega_{x, y}^{*}$. If $(x, y) \in \Omega_{x, y}^{*}$, then by (3.10) we have

$$
\begin{aligned}
\mid & F_{1}(x, y)(t)-\rho_{1} e^{\int_{0}^{t} h_{1}(s) d s} \mid \\
\leq & \left|\frac{c_{1}(t)}{1-\tau_{1}^{\prime}(t)}\right|\left|x\left(t-\tau_{1}(t)\right)\right|+\int_{t}^{\infty} \frac{e^{\int_{0}^{t} h_{1}(s) d s}}{e^{\int_{0}^{u} h_{1}(s) d s}}\left|r_{1}(u)\right|\left|x\left(u-\tau_{1}(u)\right)\right| d u \\
& +\int_{t}^{\infty} \frac{e^{\int_{0}^{t} h_{1}(s) d s}}{e^{\int_{0}^{u} h_{1}(s) d s}}\left|G_{1}\left(u, x(u), y(u), x\left(u-\tau_{1}(u)\right), y\left(u-\tau_{2}(u)\right)\right)\right| d u \\
& +\int_{t}^{\infty} \frac{e^{\int_{0}^{t} h_{1}(s) d s}}{e^{\int_{0}^{u} h_{1}(s) d s}} \int_{-\infty}^{u}\left|a_{1}(u, s)\right|\left|f_{1}(x(s), y(s))\right| d s d u, \\
\leq & \mu_{1}^{*} V^{*}+M_{1}^{*} M_{3}^{*} m_{1}^{-1} V^{*}+K_{1} M_{5}^{*} m_{1}^{-1}+M_{1}^{*} M_{1} V_{1} m_{1}^{-1},
\end{aligned}
$$

and in a similar way we have $\left|F_{2}(x, y)(t)-\rho_{2} e^{\int_{0}^{t} h_{2}(s) d s}\right| \leq \mu_{2}^{*} V^{*}+M_{2}^{*} M_{4}^{*} m_{2}^{-1} V^{*}+K_{2} M_{6}^{*} m_{2}^{-1}+M_{2}^{*} M_{2} V_{2} m_{2}^{-1}$. 
This implies that

$$
\begin{aligned}
\left|F_{1}(x, y)(t)\right| & \leq \mu_{1}^{*} V^{*}+M_{1}^{*} M_{3}^{*} m_{1}^{-1} V^{*}+K_{1} M_{5}^{*} m_{1}^{-1}+M_{1}^{*} M_{1} V_{1} m_{1}^{-1}+\rho_{1} M_{1}^{*} \\
& \leq V^{*}
\end{aligned}
$$

and

$$
\begin{aligned}
\left|F_{2}(x, y)(t)\right| & \leq \mu_{2}^{*} V^{*}+M_{2}^{*} M_{4}^{*} m_{2}^{-1} V^{*}+K_{2} M_{6}^{*} m_{2}^{-1}+M_{2}^{*} M_{2} V_{2} m_{2}^{-1}+\rho_{2} M_{2}^{*} \\
& \leq V^{*} .
\end{aligned}
$$

Hence, $F\left(\Omega_{x, y}^{*}\right) \subseteq \Omega_{x, y}^{*}$ as desired. The work to show that $A^{*}$ is completely continuous and $B^{*}$ is a contraction is similar to the corresponding work in Theorem 2.2, and hence we omit it here. Therefore, by Krasnoselskii's fixed point theorem, there exists a fixed point $(x, y) \in \Omega_{x, y}^{*}$ such that

$$
F(x, y)(t)=\left(F_{1}(x, y)(t), F_{2}(x, y)(t)\right)=(x(t), y(t))
$$

By Theorem 3.1 we know that this fixed point is a solution of (1.1).

For an arbitrary fixed point $(x, y) \in \Omega_{x, y}^{*}$ of $F$, we obtain from (3.4)-(3.7)

$$
\lim _{t \rightarrow \infty}\left|x(t)-x_{1}(t)\right|=\lim _{t \rightarrow \infty}\left|F_{1}(x, y)(t)-x_{1}(t)\right|=0,
$$

and

$$
\lim _{t \rightarrow \infty}\left|y(t)-y_{1}(t)\right|=\lim _{t \rightarrow \infty}\left|F_{2}(x, y)(t)-y_{1}(t)\right|=0
$$

By letting

$$
\begin{aligned}
x_{2}(t)= & \frac{c_{1}(t) x\left(t-\tau_{1}(t)\right)}{1-\tau_{1}^{\prime}(t)}+\int_{t}^{\infty} \frac{e^{\int_{0}^{t} h_{1}(s) d s}}{e^{\int_{0}^{u} h_{1}(s) d s}} r_{1}(u) x\left(u-\tau_{1}(u)\right) d u \\
& -\int_{t}^{\infty} \frac{e^{\int_{0}^{t} h_{1}(s) d s}}{e^{\int_{0}^{u} h_{1}(s) d s}} G_{1}\left(u, x(u), y(u), x\left(u-\tau_{1}(u)\right), y\left(u-\tau_{2}(u)\right)\right) d u \\
& -\int_{t}^{\infty} \frac{e^{\int_{0}^{t} h_{1}(s) d s}}{e^{\int_{0}^{u} h_{1}(s) d s}} \int_{-\infty}^{u} a_{1}(u, s) f_{1}(x(s), y(s)) d s d u,
\end{aligned}
$$

and

$$
\begin{aligned}
y_{2}(t)= & \frac{c_{2}(t) y\left(t-\tau_{2}(t)\right)}{1-\tau_{2}^{\prime}(t)}+\int_{t}^{\infty} \frac{e^{\int_{0}^{t} h_{2}(s) d s}}{e^{\int_{0}^{u} h_{2}(s) d s}} r_{2}(u) y\left(u-\tau_{2}(u)\right) d u \\
& -\int_{t}^{\infty} \frac{e^{\int_{0}^{t} h_{2}(s) d s}}{e^{\int_{0}^{u} h_{2}(s) d s}} G_{2}\left(u, x(u), y(u), x\left(u-\tau_{1}(u)\right), y\left(u-\tau_{2}(u)\right)\right) d u
\end{aligned}
$$




$$
-\int_{t}^{\infty} \frac{e^{\int_{0}^{t} h_{2}(s) d s}}{e^{\int_{0}^{u} h_{2}(s) d s}} \int_{-\infty}^{u} a_{2}(u, s) f_{2}(x(s), y(s)) d s d u
$$

We see that $(x, y)$ given by

$$
x(t)=x_{1}(t)+x_{2}(t), y(t)=y_{1}(t)+y_{2}(t),
$$

is an asymptotically $T$-periodic solution of (1.1). Note that by (3.4)-(3.7)

$$
\begin{aligned}
\lim _{t \rightarrow \infty}\left|x_{2}(t)\right| \leq & V^{*} \lim _{t \rightarrow \infty}\left|\frac{c_{1}(t)}{1-\tau_{1}^{\prime}(t)}\right|+V^{*} \lim _{t \rightarrow \infty} \int_{t}^{\infty} \frac{e^{\int_{0}^{t} h_{1}(s) d s}}{e^{\int_{0}^{u} h_{1}(s) d s}}\left|r_{1}(u)\right| d u \\
& +K_{1} \lim _{t \rightarrow \infty} \int_{t}^{\infty} \frac{e^{\int_{0}^{t} h_{1}(s) d s}}{e^{\int_{0}^{u} h_{1}(s) d s}} d u \\
& +M_{1} \lim _{t \rightarrow \infty} \int_{t}^{\infty} \frac{e^{\int_{0}^{t} h_{1}(s) d s}}{e^{\int_{0}^{u} h_{1}(s) d s}} \int_{-\infty}^{u}\left|a_{1}(u, s)\right| d s d u
\end{aligned}
$$

Hence

$$
\lim _{t \rightarrow \infty} x_{2}(t)=0
$$

Similarly

$$
\lim _{t \rightarrow \infty} y_{2}(t)=0
$$

Finally, we show that $x_{1}$ and $y_{1}$ are $T$-periodic. From (3.1), one can see

$$
\begin{aligned}
x_{1}(t+T) & =c_{1} e^{\int_{0}^{t+T} h_{1}(s) d s} \\
& =c_{1} e^{\int_{0}^{t} h_{1}(s) d s} e^{\int_{t}^{t+T} h_{1}(s) d s} \\
& =c_{1} e^{\int_{0}^{t} h_{1}(s) d s} e^{\int_{0}^{T} h_{1}(s) d s} \\
& =c_{1} e^{\int_{0}^{t} h_{1}(s) d s} \\
& =x_{1}(t) .
\end{aligned}
$$

Similarly, $y_{1}$ is $T$-periodic.

Data Availability. No data were used to support this study.

Conflict of interest. The authors declare no conflict of interest. 


\section{References}

1. A. Ardjouni and A. Djoudi, Existence of periodic solutions for a second order nonlinear neutral differential equation with functional delay, Electronic Journal of Qualitative Theory of Differential Equations, 31 (2012), 1-9.

2. A. Ardjouni and A. Djoudi, Existence of periodic solutions for a second-order nonlinear neutral differential equation with variable delay, Palestine Journal of Mathematics, $\mathbf{3}$ (2014), No.2, 191-197.

3. A. Ardjouni and A. Djoudi, Existence of positive periodic solutions for a nonlinear neutral differential equations with variable delay, Applied Mathematics E-Notes, 12 (2012), 94-101.

4. A. Ardjouni and A. Djoudi, Periodic solutions in totally nonlinear dynamic equations with functional delay on a time scale, Rend. Sem. Mat. Univ. Politec. Torino, 68 (2010), No.4, 349-359.

5. A. Ardjouni, A. Djoudi and I. Soualhia, Stability for linear neutral integro-differential equations with variable delays, EJDE, 172 (2012), 1-14.

6. E. Biçer and C, Tunç, On the existence of periodic solutions to non-linear neutral differential equations of first order with multiple delays, Proceedings of the Pakistan Academy of Sciences, 52 (2015), No. 1, 89-94.

7. T. A. Burton, Volterra Integral and Differential Equations, Academic Press, New York, 1983.

8. A. Caicedo, C. Cuevas and H. R. Henriquez, Asymptotic periodicity for a class of partial integro-differential equations, ISRN Mathematical Analysis, 2011 (2011), 118.

9. J. M. Cushing, Forced asymptotically periodic solutions of predator-prey systems with or without hereditary effects, SIAM Journal on Applied Mathematics, 30 (1976), No. 4, 665-674.

10. J. M. Cushing, Integro-differential Equations and Delay Models in Population Dynamics, Lecture Notes in Biomathematics 20, Springer, New York, 1977.

11. J. A. Hudson, The Excitation and Propagation of Elastic Waves, Cambridge Monographs on Mechanics and Applied Mathematics, Cambridge University Press, Cambridge-New York, 1980.

12. H. R. Henriquez, C. Cuevas and A. Caicedo, Asymptotically periodic solutions of neutral partial differential equations with infinite delay, Communications on Pure and Applied Analysis, 12 (2013), No. 5, 2031-2068.

13. M. N. Islam, Asymptotically periodic solutions of Volterra integral equations, Electronic Journal of Differential Equations, 83 (2016), 1-9.

14. G. Leugering, A generation result for a class of linear thermoviscoelastic material, Dynamical problems in mathematical physics (Oberwolfach, 1982), 107-117, Methoden Verfahren Math. Phys., 26, Lang, Frankfurt am Main, 1983. 
15. J. Liang and T. J. Xiao, Semilinear integro-differential equations with nonlocal initial condit ions, Comput. Math. Appl., 47 (2004), no. 6-7, 863-875.

16. J. Liang, T. J. Xiao and J. Van Casteren, A note on semilinear abstract functional differential and integro-differential equations with infinite delay, Appl. Math. Lett., 17 (2004), No. 4, 473-477.

17. Y. Li and G. Xu, Positive periodic solutions for an integro-differential model of mutualism, Applied Mathematics Letters, 14 (2001), 525-530.

18. R. C. MacCamy, An integro-differential equation with application in heatow, Quart. Appl. Math., 35(1977/78), No.1, 1-19.

19. B. Mansouri, A. Ardjouni and A. Djoudi, Existence of positive periodic solutions for two types of third-order nonlinear neutral differential equations with variable coefficients, Differential Equations and Control Processes, 3 (2018), 46-63.

20. B. Mansouri, A. Ardjouni and A. Djoudi, Periodicity and stability in neutral nonlinear differential equations by Krasnoselskii's fixed point theorem, CUBO A Mathematical Journal, 19 (2017), No. 03, 15-29.

21. R. K. Miller, An integro-differential equation for rigid heat conductors with memory, J. Math. Anal. Appl., 66 (1978), No.2, 313-332.

22. Y. N. Raffoul, Analysis of periodic and asymptotically periodic solutions in nonlinear coupled Volterra integro-differential systems, Turk. J. Math., 42 (2018), 108-120.

23. Y. N. Raffoul, Stability in neutral nonlinear differential equations with functional delays using fixed-point theory, Math. Comput. Modelling, 40 (2004), 691-700.

24. Di R. Smart, Fixed Point Theorems, Cambridge Univ. Press, Cambridge, 1980.

25. V. Volterra, Sur la théorie mathématique des phénomènes héréditaires, J. Math. Pur. Appl., 7 (1928), 249-298.

26. F. Y. Wei and K. Wang, Global stability and asymptotically periodic solutions for nonautonomous cooperative Lotka-Volterra diffusion system, Applied Mathematics and Computation, 182 (2006), No. 1, 161-165.

27. Z. J. Zeng, Asymptotically periodic solution and optimal harvesting policy for Gompertz system, Nonlinear Analysis: Real World Applications, 12 (2011), No. 3, 14011409. 